\title{
Constraints on the diffusive shock acceleration from the nonthermal X-ray thin shells in SN 1006 NE rim
}

\author{
R. Yamazaki ${ }^{1}$, T. Yoshida ${ }^{2}$, T. Terasawa ${ }^{3}$, A. Bamba ${ }^{1}$, and K. Koyama ${ }^{1}$ \\ ${ }^{1}$ Department of Physics, Kyoto University, Kyoto 606-8502, Japan \\ e-mail: yamazaki@tap.scphys.kyoto-u.ac.jp , bamba@cr.scphys . kyoto-u.ac.jp, \\ koyama@cr.scphys.kyoto-u.ac.jp \\ ${ }^{2}$ Faculty of Science, Ibaraki University, Mito 310-8512, Japan \\ e-mail: yoshidat@mx . ibaraki .ac .jp \\ 3 Earth \& Planetary Science, Graduate School of Science, University of Tokyo, 7-3-1 Hongo, Bunkyo-ku, 113 Tokyo, Japan \\ e-mail: terasawa@eps.s.u-tokyo.ac.jp
}

Received 20 August 2003 / Accepted 6 November 2003

\begin{abstract}
Characteristic scale lengths of nonthermal X-rays from the SN 1006 NE rim, which are observed by Chandra, are interpreted in the context of diffusive shock acceleration with the assumption that the observed spatial profile of nonthermal $\mathrm{X}$-rays corresponds to that of accelerated electrons with energies of a few tens of TeV. To explain the observed scale lengths, we construct two simple models with a test particle approximation, where the maximum energy of accelerated electrons is determined by the age of SN 1006 (age-limited model) or the energy loss (energy loss-limited model), and constrain the magnetic field configuration and the diffusion coefficients of accelerated electrons. When the magnetic field is nearly parallel to the shock normal, the magnetic field should be in the range of 20-85 $\mu \mathrm{G}$ and highly turbulent both upstream and downstream, which means that the mean free path of accelerated electrons is of the order of their gyro-radius (Bohm limit). This situation can be realized both in the age-limited and energy loss-limited model. On the other hand, when the magnetic field is nearly perpendicular to the shock normal, which can exist only in the age-limited case, the magnetic field is several $\mu \mathrm{G}$ upstream and 14-20 $\mu \mathrm{G}$ downstream, and the upstream magnetic field is less turbulent than the downstream.
\end{abstract}

Key words. acceleration of particles - ISM: supernova remnants - X-rays: individual: SN 1006

\section{Introduction}

Galactic cosmic rays with energies of less than $10^{15.5} \mathrm{eV}$ (the "knee" energy) are commonly believed to be generated by supernova remnants (SNRs). SN 1006 is one of the SNRs thought to be an accelerator of such high energy particles. Koyama et al. (1995) discovered synchrotron X-rays from the rim of this $\mathrm{SNR}$, indicating the existence of accelerated electrons with an energy of more than a few tens of $\mathrm{TeV}$. The detection of $\mathrm{TeV}$ $\gamma$-rays from the northeastern (NE) rim of SN 1006 (Tanimori et al. 1998) implies the presence of high energy particles, since $\mathrm{TeV} \gamma$-rays arise from the Inverse Compton (IC) process, in which cosmic microwave background (CMB) photons are upscattered by high energy electrons (Tanimori et al. 2001), or the hadronic process, in which $\pi^{0}$ particles made by collisions between accelerated and interstellar protons decay into $\gamma$-ray photons (Berezhko et al. 2002; Aharonian \& Atoyan 1999).

The mechanism for cosmic ray acceleration has also been studied for a long time and the most plausible process is a diffusive shock acceleration (DSA) (Bell 1978;

Send offprint requests to: R. Yamazaki,

e-mail: yamazaki@tap.scphys.kyoto-u.ac.jp
Blandford \& Ostriker 1978; Drury 1983; Blandford \& Eicher 1987; Jones \& Ellison 1991; Malkov \& Drury 2001). Many authors have explained the observed properties of SN 1006 in the context of the DSA but the conclusions are different due to arbitrary assumptions for unknown physical parameters, such as the magnetic field configuration, the diffusion coefficient, the injection rate, and the electron to proton ratio (Ellison et al. 2000; Berezhko et al. 2002; Völk et al. 2003; Aharonian \& Atoyan 1999; Reynolds 1998; Dyer et al. 2001; Allen et al. 2001; Achterberg et al. 1998). For example, at present the origin of $\mathrm{TeV} \gamma$-rays can be explained by both leptonic and hadronic models. This comes from an insufficient theoretical understanding; apart from a globally successful picture of the DSA, detailed but important processes, such as the injection or the reflection of accelerated particles that determine the above unknown quantities, are not well understood. Worse yet, previous observations in the hard X-ray band had insufficient spatial resolution to resolve small-scale structures near the shock front, and could not strongly constrain the theoretical parameters.

Recently, Bamba et al. (2003a,b) reported results for spectral and spatial studies of thermal and non-thermal shock structure in the NE rim of SN 1006 with the excellent spatial 
resolution of Chandra. Similar results are also reported by Long et al. (2003) with Chandra data. Bamba et al. (2003a) estimated the scale length of thermal and non-thermal X-rays upstream and downstream of the shock front, which means that the direct measurement of the diffusion coefficients has become possible.

In this paper, we show that the important physical parameters for the magnetic field in the acceleration site can be constrained by the spatial distribution of observed nonthermal X-rays. Section 2 summarized results of data analyses by Bamba et al. (2003a). We construct two models in Sect. 3; one assumes that the maximum energy of accelerated electrons is determined by the age of SNR, while the other is by the energy loss process such as synchrotron or IC cooling. Finally, Sect. 4 is devoted to the discussion of the validity of our estimation. Throughout the paper, indices " $u$ " and " $d$ " represent upstream and downstream, respectively.

\section{Observed properties of the nonthermal NE shell}

We used the Chandra archival data of the ACIS ${ }^{1}$, which has the spatial resolution of $0.5 \mathrm{arcsec}$, on the NE shell of SN 1006 (Observation ID = 00732) observed on July 10-11, 2000 with the targeted position at $(\mathrm{RA}, \mathrm{Dec})=\left(15^{\mathrm{h}} 03^{\mathrm{m}} 51^{\mathrm{s}} \cdot 6\right.$, $-41^{\mathrm{d}} 51^{\mathrm{m}} 18.8$ ) in $\mathrm{J} 2000$ coordinates. Bamba et al. (2003a,b) and Long et al. (2003) report that there are very thin filaments in the hard X-ray band image, which must be the acceleration sites of high energy electrons. Details of the observation and the analysis are found in these papers.

As shown in Fig. 2 of Bamba et al. (2003a), they made profiles of six filaments and found that the upstream scale length $w_{\mathrm{u}}$ ranges between 0.01 and $0.1 \mathrm{pc}$, while the downstream scale length $w_{\mathrm{d}}$ varies from 0.06 to $0.4 \mathrm{pc}$ using the exponential function with an adopted distance of $2.18 \mathrm{kpc}$ (Winkler et al. 2003). The mean values of $w_{\mathrm{u}}$ and $w_{\mathrm{d}}$ are $0.05 \mathrm{pc}$ and $0.2 \mathrm{pc}$, respectively.

Bamba et al. (2003a) also fitted the X-ray spectra for the six filaments with an srcut model in the XSPEC package (Reynolds 1998; Reynolds \& Keohane 1999). The radio spectral index of 0.57 was adopted from the result of Allen et al. (2001). As a result, the best-fit roll-off frequency $v_{\text {rolloff }}=2.6$ $(1.9-3.3) \times 10^{17} \mathrm{~Hz}$ was derived. The quantity $v_{\text {rolloff }}$ is written in terms of magnetic field $(B)$ and the maximum energy of accelerated electrons $\left(E_{\max }\right)$ as (Reynolds \& Keohane 1999)

$v_{\text {rolloff }}=5 \times 10^{17} \mathrm{~Hz}\left(\frac{B}{10 \mu \mathrm{G}}\right)\left(\frac{E_{\max }}{100 \mathrm{TeV}}\right)^{2}$.

Since most of the nonthermal X-ray photons are observed downstream, the synchrotron radiation is mainly due to the downstream region. Therefore, it is possible to adopt $B$ in Eq. (1) with the downstream magnetic field $B_{\mathrm{d}}$.

\section{Interpretation of the observed width of nonthermal X-ray filaments}

Two simple models are considered in the context of DSA with a test-particle approximation. We assume that the spatial

\footnotetext{
${ }^{1}$ http://asc.harvard.edu/proposer/POG/html/ACIS.html
}

distribution of nonthermal X-rays coincides with that of the accelerated electrons to the maximum energy, while thermal $\mathrm{X}$-rays trace the spatial profile of a background plasma and hence a magnetic field. For a steady state, there is no spatial structure of accelerated particles downstream (Blandford \& Ostriker 1978). However, one should consider the finite-time or energy-loss effect, which makes the spatial profile in the downstream region.

For simplicity, we assume magnetic fields are spatially uniform both upstream and downstream at least in the nonthermal X-ray emitting region. Since the fraction of magnetic pressure to ram pressure is estimated as $\left(B^{2} / 8 \pi\right) /\left(m_{\mathrm{H}} n u_{\mathrm{s}}^{2}\right) \sim$ $2 \times 10^{-5}(B / 10 \mu \mathrm{G})^{2}$, where we assume the number density of thermal plasma $n \sim 1 \mathrm{~cm}^{-3}$ and the shock velocity $u_{\mathrm{s}} \sim$ $3 \times 10^{3} \mathrm{~km} \mathrm{~s}^{-1}$, the magnetic pressure does not affect the dynamics of SNR. We therefore can adopt the self-similar solution derived by Ratkiewicz et al. (1994). Our assumption of a spatially uniform magnetic field is a good approximation in the narrow range around the shock front.

Since the wide-band spectrum shows a break on the X-ray band, electrons accelerated near the maximum energy $E_{\max }$ contribute to nonthermal X-ray emission. The quantity $E_{\max }$ is determined by the age of the SNR or by the balance of the acceleration and the energy-loss efficiencies. To discuss this, we consider three time scales; the acceleration time scale $t_{\text {acc }}$, the energy loss time scale $t_{\text {loss }}$, and the age of SN $1006 t_{\text {age }}$. The energy loss of high energy electrons can be neglected when $t_{\mathrm{acc}}<t_{\text {loss }}$. Then, $E_{\max }$ is determined by $t_{\mathrm{acc}}=t_{\mathrm{age}}$. On the other hand, $E_{\max }$ is limited by the energy loss if $t_{\mathrm{acc}}=t_{\text {loss }}<t_{\text {age }}$. We investigate these two cases separately in the following subsections.

The acceleration time is given by Drury (1983) as

$t_{\mathrm{acc}}=\frac{3}{u_{\mathrm{u}}-u_{\mathrm{d}}}\left(\frac{K_{\mathrm{u}}}{u_{\mathrm{u}}}+\frac{K_{\mathrm{d}}}{u_{\mathrm{d}}}\right)$

where $u$ and $K$ are the velocity of bulk flow in the shock frame and the diffusion coefficients for accelerated electrons at the maximum energy, respectively. The diffusion coefficients are given by quasi-linear theory (Skilling 1975; Blandford \& Eichler 1987). Let the mean free path of accelerated electrons parallel to the magnetic field be a constant factor $\eta$ times the gyro radius $r_{\mathrm{g}}=E /(\mathrm{e} B)$ both upstream and downstream. Then the diffusion coefficients in upstream and downstream regions can be written in terms of $\eta_{\mathrm{u}}, \eta_{\mathrm{d}}$, and the angle between the upstream magnetic field and the shock normal $\theta$ (Jokipii 1987),

$K_{\mathrm{u}}=\frac{c E_{\max }}{3 \mathrm{e} B_{\mathrm{u}}} \eta_{\mathrm{u}}\left(\cos ^{2} \theta+\frac{\sin ^{2} \theta}{1+\eta_{\mathrm{u}}^{2}}\right)$,

$$
\begin{aligned}
K_{\mathrm{d}}=\frac{c E_{\mathrm{max}}}{3 \mathrm{e} B_{\mathrm{d}}} \eta_{\mathrm{d}}\left(\cos ^{2} \theta\right. & \left.+r^{2} \sin ^{2} \theta\right)^{-1} \\
& \times\left(\cos ^{2} \theta+\frac{r^{2} \sin ^{2} \theta}{1+\eta_{\mathrm{d}}^{2}}\right),
\end{aligned}
$$

where $r$ is the compression ratio. In this paper, since we assume that the shock is sufficiently strong and that shock structure is 

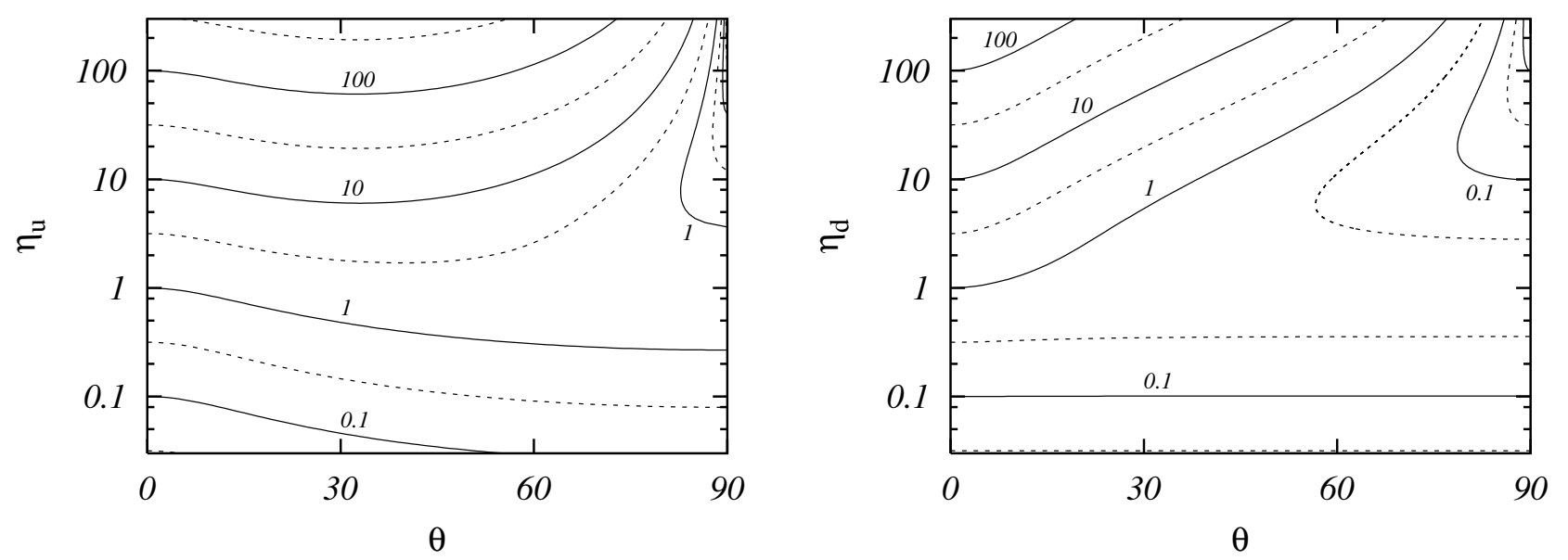

Fig. 1. Contour maps of the functions $f\left(\eta_{\mathrm{u}}, \theta\right)$ (left panel) and $g\left(\eta_{\mathrm{d}}, \theta\right)$ (right).

not affected by the cosmic-ray pressure, $r$ should be 4 and the upstream and downstream magnetic field are related as

$\frac{B_{\mathrm{d}}}{B_{\mathrm{u}}}=R(\theta):=\left(\cos ^{2} \theta+r^{2} \sin ^{2} \theta\right)^{\frac{1}{2}}$.

Upstream and downstream velocities are related as $u_{\mathrm{u}}=r u_{\mathrm{d}} \equiv$ $u_{\mathrm{s}}$, and throughout the paper, we adopt $u_{\mathrm{s}}=2.89 \times 10^{3} \mathrm{~km} \mathrm{~s}^{-1}$ (Ghavamian et al. 2002). It is convenient to rewrite diffusion coefficients using Eqs. (3)-(5) as

$K_{\mathrm{u}}=\frac{c E_{\max }}{3 \mathrm{e} B_{\mathrm{d}}} f\left(\eta_{\mathrm{u}}, \theta\right)$

$K_{\mathrm{d}}=\frac{c E_{\max }}{3 \mathrm{e} B_{\mathrm{d}}} g\left(\eta_{\mathrm{d}}, \theta\right)$,

where $f\left(\eta_{\mathrm{u}}, \theta\right)$ and $g\left(\eta_{\mathrm{d}}, \theta\right)$ are given by

$f\left(\eta_{\mathrm{u}}, \theta\right)=\eta_{\mathrm{u}}\left(\cos ^{2} \theta+r^{2} \sin ^{2} \theta\right)^{1 / 2}\left(\cos ^{2} \theta+\frac{\sin ^{2} \theta}{1+\eta_{\mathrm{u}}^{2}}\right)$,

$g\left(\eta_{\mathrm{d}}, \theta\right)=\eta_{d}\left(\cos ^{2} \theta+r^{2} \sin ^{2} \theta\right)^{-1}\left(\cos ^{2} \theta+\frac{r^{2} \sin ^{2} \theta}{1+\eta_{\mathrm{d}}^{2}}\right)$.

Note that the downstream magnetic field $B_{\mathrm{d}}$ is used both in Eqs. (6) and (7). Figure 1 shows the contour plots of $f\left(\eta_{\mathrm{u}}, \theta\right)$ and $g\left(\eta_{\mathrm{d}}, \theta\right)$. If $\theta \gtrsim 83^{\circ}, f$ can be smaller than unity in the case of $\eta_{\mathrm{u}}>1$. The quantity $g$ is smaller than 0.1 for $\eta_{\mathrm{d}}>1$ and $\theta \gtrsim 79^{\circ}$

Next, we consider the energy loss time scale. Two processes cause the energy loss of electrons: synchrotron radiation and the IC effect by cosmic microwave background photons. In the following calculations, the latter process can be neglected. Then, we can simply write

$$
\begin{aligned}
t_{\text {loss }} & =\frac{6 \pi m_{\mathrm{e}}^{2} c^{3}}{\sigma_{T} E B^{2}} \\
& =1.25 \times 10^{3} \mathrm{yrs}\left(\frac{E_{\max }}{100 \mathrm{TeV}}\right)^{-1}\left(\frac{B}{10 \mu \mathrm{G}}\right)^{-2} .
\end{aligned}
$$

When one compares $t_{\text {loss }}$ with $t_{\text {acc }}$, the mean value of the magnetic field that accelerated electrons experience should be adopted. We can estimate the mean magnetic field as

$\left\langle B^{2}\right\rangle=\alpha B_{\mathrm{u}}^{2}+(1-\alpha) B_{\mathrm{d}}^{2}=\chi B_{\mathrm{d}}^{2}$ where $\alpha=\Delta t_{\mathrm{u}} /\left(\Delta t_{\mathrm{u}}+\Delta t_{\mathrm{d}}\right)$ is the time fraction that accelerating electrons are upstream. For the particles in the acceleration process, we can estimate $\Delta t_{\mathrm{u}} / \Delta t_{\mathrm{d}} \sim\left(K_{\mathrm{u}} / u_{\mathrm{u}}\right) /\left(K_{\mathrm{d}} / u_{\mathrm{d}}\right)=f /(r g)$ from Eqs. (6) and (7). Then, we obtain $\chi=\left(R^{-2} f+r g\right) /(f+r g)$, which ranges between 0 and 1, where $R$ is defined in Eq. (5).

\subsection{Age-limited case}

First, we investigate the case in which the acceleration time is nearly equal to the age of SN $1006 t_{\text {acc }} \sim t_{\text {age }}$. This condition implies that the observed nonthermal X-rays are emitted by electrons that have been accelerated up to now.

The particles in the diffusive shock acceleration process are transported upstream by the diffusion, and are advected downstream. The diffusion and advection time scales to move a scale-length $w$ are $t_{\mathrm{adv}}=w / u$ and $t_{\mathrm{dif}}=w^{2} / K$. In the upstream region, the accelerated particles can reach the point where the advection is balanced by diffusion, i.e. $t_{\mathrm{adv}}=t_{\mathrm{dif}}$. Therefore, the observed upstream-width of nonthermal X-rays can be written as

$w_{\mathrm{u}}=\frac{K_{\mathrm{u}}}{u_{\mathrm{u}}}=\frac{c E_{\max }}{3 \mathrm{e} B_{\mathrm{d}} u_{\mathrm{s}}} f\left(\eta_{\mathrm{u}}, \theta\right)$.

In the downstream region, if the particles are advected too far from the shock front, they cannot return upstream to obtain further energy. Therefore, the particles being accelerated should stay in the region where $t_{\text {dif }}$ is smaller than $t_{\text {adv }}$. Thus, we obtain the same equation as upstream,

$w_{\mathrm{d}}=\frac{K_{\mathrm{d}}}{u_{\mathrm{d}}}=\frac{c E_{\max }}{3 \mathrm{e} B_{\mathrm{d}} u_{\mathrm{s}}} r g\left(\eta_{\mathrm{d}}, \theta\right)$.

Then, Eq. (2) becomes

$t_{\mathrm{acc}}=\frac{3 r}{r-1} \frac{w_{\mathrm{u}}+w_{\mathrm{d}}}{u_{\mathrm{s}}} \sim 3.4 \times 10^{2} \mathrm{yrs}\left(\frac{w_{\mathrm{u}}+w_{\mathrm{d}}}{0.25 \mathrm{pc}}\right)$.

Since $t_{\text {acc }}$ is comparable to the age of the SN 1006, our assumption $t_{\text {acc }} \sim t_{\text {age }}$ is justified. Eliminating $E_{\max }$ with Eq. (1), we can rewrite (12) and (13) as

$w_{\mathrm{u}}=0.27 \mathrm{pc}\left(\frac{v_{\text {rolloff }}}{2.6 \times 10^{17} \mathrm{~Hz}}\right)^{\frac{1}{2}}\left(\frac{B_{\mathrm{d}}}{10 \mu \mathrm{G}}\right)^{-\frac{3}{2}} f\left(\eta_{\mathrm{u}}, \theta\right)$, 

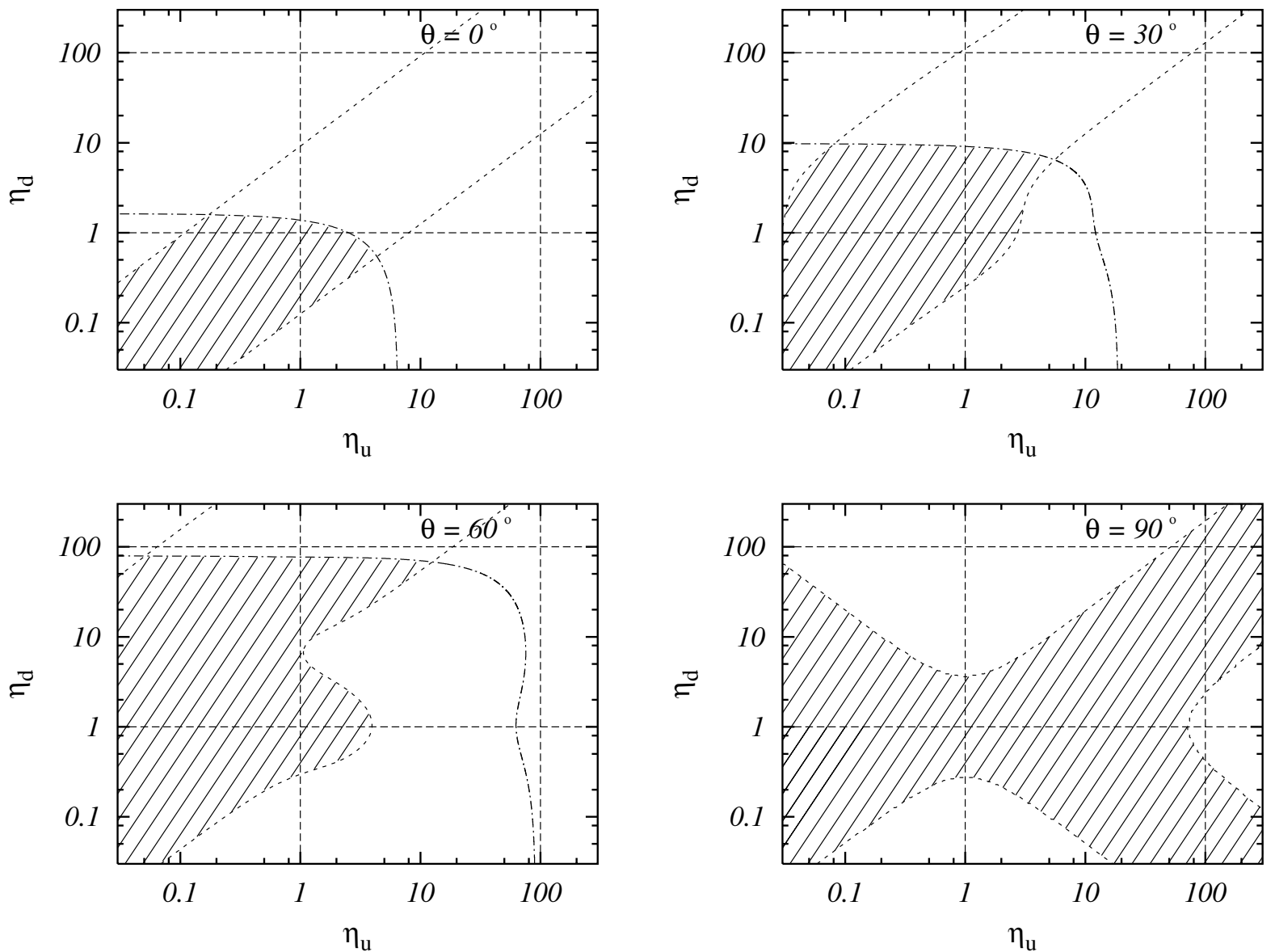

Fig. 2. Points in the shaded regions surrounded by dotted and dot-dashed lines satisfy Eqs. (1), (5), (12), and (13) for fixed $\theta$ in the age-limited case. Dotted lines represent Eq. (A.1) with $w_{\mathrm{d}} / w_{\mathrm{u}}=0.6$ or 40 , while dot-dashed lines Eq. (A.4) with $v_{\text {rolloff }}=1.9 \times 10^{17} \mathrm{~Hz}$, the region above which is forbidden since $t_{\text {acc }}>t_{\text {loss }}$.

$w_{\mathrm{d}}=1.1 \mathrm{pc}\left(\frac{v_{\text {rolloff }}}{2.6 \times 10^{17} \mathrm{~Hz}}\right)^{\frac{1}{2}}\left(\frac{B_{\mathrm{d}}}{10 \mu \mathrm{G}}\right)^{-\frac{3}{2}} g\left(\eta_{\mathrm{d}}, \theta\right)$,

respectively.

Let us take $w_{\mathrm{u}} \sim 0.05 \mathrm{pc}, w_{\mathrm{d}} \sim 0.2 \mathrm{pc}$, and $v_{\text {rolloff }} \sim 2.6 \times$ $10^{17} \mathrm{~Hz}$ as typical observed quantities (Bamba et al. 2003a). Then, Eqs. (15) and (16) give $B_{\mathrm{d}} \sim 30 f^{2 / 3} \mu \mathrm{G} \sim 30 g^{2 / 3} \mu \mathrm{G}$. In the case of $\theta \sim 0^{\circ}$, the value of $B_{\mathrm{d}}$ can be determined as follows. We see that since $\eta_{\mathrm{u}} \geq 1, f$ should be greater than $\sim 1$, i.e. $B_{\mathrm{d}} \gtrsim 30 \mu \mathrm{G}$. Independently of this, the condition $t_{\text {acc }} \lesssim t_{\text {loss }}$ gives the upper limit of $B_{\mathrm{d}}$ as $B_{\mathrm{d}} \lesssim 30 \mu \mathrm{G}$ (see Eq. (A.5)). Therefore, we obtain $B_{\mathrm{d}} \sim 30 \mu \mathrm{G}$. This argument has been used in Bamba et al. (2003b). On the other hand, as we have mentioned before, cases of $f \lesssim 1$ can be realized if $\theta \gtrsim 80^{\circ}$, i.e. the magnetic field is nearly perpendicular to the shock normal. Then $B_{\mathrm{d}}$ may be smaller than $\sim 30 \mu \mathrm{G}$.

Let us vary the observed quantities $w_{\mathrm{u}}, w_{\mathrm{d}}$, and $v_{\text {rolloff }}$ in the range of the observed errors (at a $90 \%$ confidence level). We have six unknown parameters $E_{\max }, B_{\mathrm{u}}, B_{\mathrm{d}}, \eta_{\mathrm{u}}$, $\eta_{\mathrm{d}}$, and $\theta$. Conditions (1), (5), (12), (13), and $t_{\text {acc }} \lesssim t_{\text {loss }}$ are used to relate these quantities. Details are summarized in the Appendix A. Figure 2 shows the allowed regions of $\eta_{\mathrm{u}}$ or $\eta_{\mathrm{d}}$ for fixed $\theta$. When we take $\theta=0^{\circ}$, the case of $\eta_{\mathrm{u}}=\eta_{\mathrm{d}}=1$ (Bohm limit both upstream and downstream) is marginally acceptable, since $\eta$ should satisfy $1 \leq \eta \lesssim c / u_{\mathrm{s}} \sim 10^{2}$ (Jokipii 1987). Then the magnetic field has values of $B_{\mathrm{u}}=B_{\mathrm{d}}=20-$ $78 \mu \mathrm{G}$. Equation (A.5) shows that the maximum value of magnetic fields is achieved when the observed quantities $v_{\text {rolloff }}, w_{\mathrm{u}}$, and $w_{\mathrm{d}}$ have the minimum values. On the other hand, when $\theta \gtrsim 85^{\circ}$, small magnetic fields are possible. If we choose $w_{\mathrm{u}}=0.1 \mathrm{pc}, w_{\mathrm{d}}=0.3 \mathrm{pc}$, and $v_{\text {rolloff }}=2 \times 10^{17} \mathrm{~Hz}$, then $\eta_{\mathrm{u}} \sim 10$ and $\eta_{\mathrm{d}} \sim 1$, the magnetic fields are $B_{\mathrm{d}} \sim 4 B_{\mathrm{u}} \sim 14-$ $20 \mu \mathrm{G}$. This result has been suggested by Bamba et al. (2003a).

The left panel of Fig. 4 represents the allowed region of $E_{\max }$ and $B_{\mathrm{d}}$ (see Eqs. (A.6) and (A.7)). The solid lines describe Eq. (1) with $v_{\text {rolloff }}=(1.9-3.3) \times 10^{17} \mathrm{~Hz}$, while the dashed lines a boundary of the region in which a gyro-radius of accelerated electrons in the downstream $r_{\mathrm{g}}=E_{\max } /\left(\mathrm{e} B_{\mathrm{d}}\right) \propto$ $g^{-1} w_{\mathrm{d}}$. In order to satisfy $1 \leq \eta \leq 10^{2}$ and all of the other conditions, the quantity $g^{-1}\left(w_{\mathrm{d}} / \mathrm{pc}\right)$ should range between 0.09 and 27.

\subsection{Energy loss-limited case}

If the maximum energy of accelerated electrons $E_{\max }$ is determined by

$t_{\mathrm{acc}}=t_{\mathrm{loss}}$, 

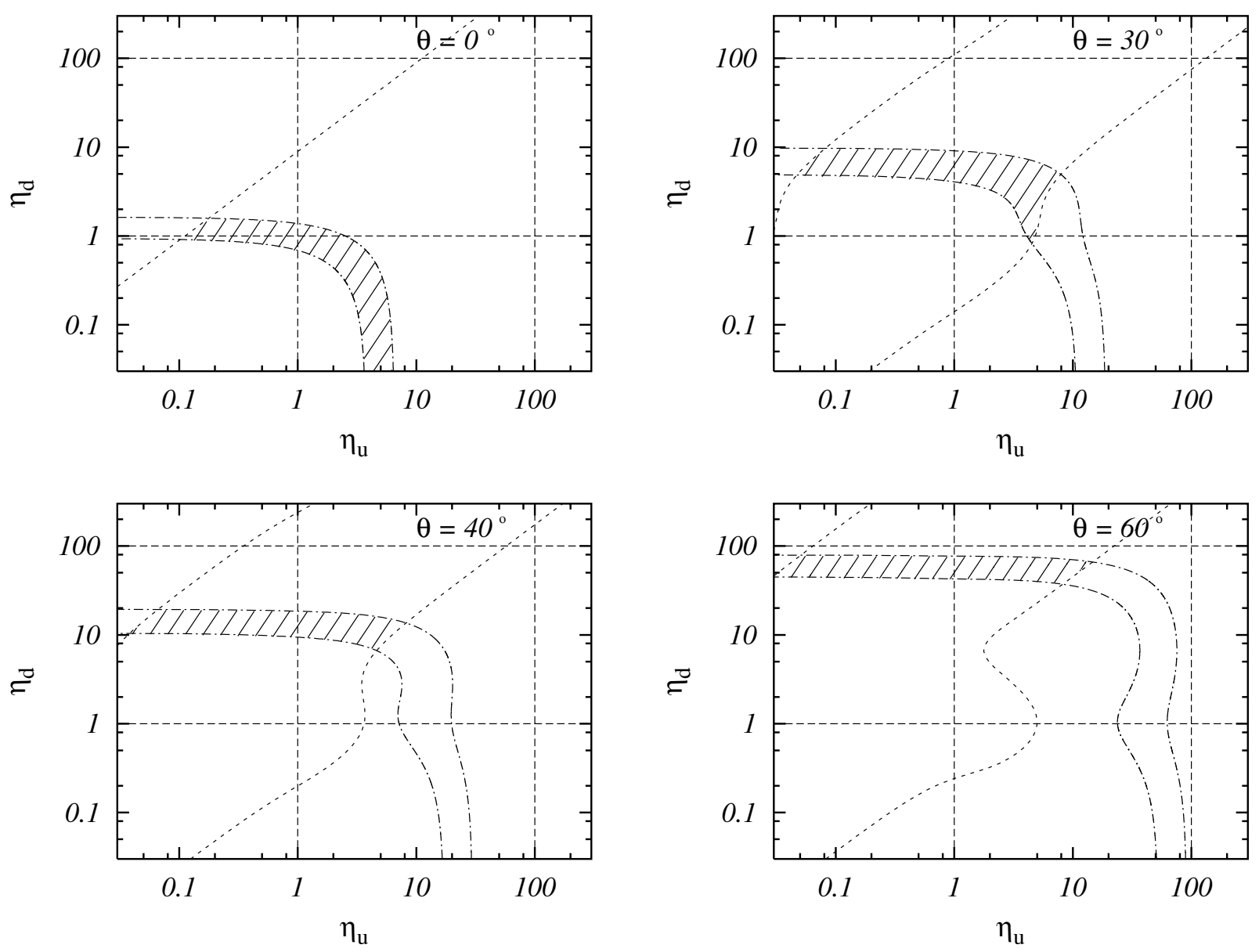

Fig. 3. Points in the shaded regions surrounded by dotted and dot-dashed lines satisfy Eqs. (1), (5), (17), (18), (19), and $t_{\text {loss }}<t_{\text {age }}$ for fixed $\theta$ in the energy loss-limited case. Dotted lines represent Eq. (A.14) with $w_{\mathrm{d}} / w_{\mathrm{u}}=0.6$ or 40 , while dot-dashed lines Eq. (A.13) with $v_{\text {rolloff }}=1.9 \times 10^{17}$ or $3.3 \times 10^{17} \mathrm{~Hz}$.

the motion of accelerated particles toward the upstream region might be obstructed by the energy loss effect as well as the advection. Let us consider in the upstream region, the energy loss time scale $t_{\text {cool }}{ }^{2}$ in addition to the advection time scale $t_{\mathrm{adv}}=w_{\mathrm{u}} / u_{\mathrm{u}}$ and the diffusion time scale $t_{\mathrm{dif}}=w_{\mathrm{u}}^{2} / K_{\mathrm{u}}$. If $t_{\mathrm{dif}}=t_{\mathrm{adv}}<t_{\text {cool }}$, the observed width of nonthermal X-rays in the upstream is given by $w_{\mathrm{u}}=K_{\mathrm{u}} / u_{\mathrm{u}}$ as well as the agelimited case, while in the case of $t_{\mathrm{dif}}=t_{\mathrm{cool}}<t_{\mathrm{adv}}, w_{\mathrm{u}}$ is given by $w_{\mathrm{u}}=\left(K_{\mathrm{u}} t_{\mathrm{cool}}\right)^{\frac{1}{2}}$. Therefore, we can write

$w_{\mathrm{u}}=\min \left\{K_{\mathrm{u}} / u_{\mathrm{u}},\left(K_{\mathrm{u}} t_{\text {cool }}\right)^{\frac{1}{2}}\right\}$.

On the other hand, the observed scale length of nonthermal $\mathrm{X}$-ray filaments in the downstream $w_{\mathrm{d}}$ is determined by the cooling time scale as

$w_{\mathrm{d}}=\max \left\{u_{\mathrm{d}} t_{\text {cool }},\left(K_{\mathrm{d}} t_{\text {cool }}\right)^{\frac{1}{2}}\right\}$.

We can now use five equations (1), (5), (17), (18), and (19) for six unknown parameters $E_{\max }, B_{\mathrm{u}}, B_{\mathrm{d}}, \eta_{\mathrm{u}}, \eta_{\mathrm{d}}$, and $\theta$, and solve these equations with fixed $\theta$ under the condition of $t_{\text {loss }}<t_{\text {age }}$. We summarize the detailed calculation procedure in Appendix A.

\footnotetext{
${ }^{2}$ To avoid confusion, here we use the notation " $t_{\text {cool }}$ " which is compared with $t_{\text {adv }}$ or $t_{\text {dif }}$, in order to distinguish " $t_{\text {loss }}$ " used when one compares with $t_{\text {acc }}$ or $t_{\text {age }}$.
}

Figure 3 shows the results for individual $\theta$. We consider, as well as the age-limited case, the errors associated with the analysis of Bamba et al. (2003a). When $\theta=0^{\circ}$, the case of $\eta_{\mathrm{u}}=\eta_{\mathrm{d}}=1$ is again acceptable. Then, the magnetic field is in the range of $B_{\mathrm{u}}=B_{\mathrm{d}}=23-85 \mu \mathrm{G}$. If $\theta \lesssim 30^{\circ}$, the downstream magnetic field can be in the Bohm limit $\eta_{\mathrm{d}}=1$, then $\eta_{\mathrm{u}} \sim 1-8$ and $B_{\mathrm{d}} \sim 23-85 \mu \mathrm{G}$. However, if $\theta$ is larger than $\sim 35^{\circ}$, then $\eta_{\mathrm{d}} \gtrsim 10$ and $\eta_{\mathrm{u}} \lesssim 10$. This implies that the upstream magnetic field is more turbulent than the downstream, which seems to be unrealistic.

Indeed, as shown in A.2, scale lengths are given by $w_{\mathrm{u}}=$ $K_{\mathrm{u}} / u_{\mathrm{u}}$ and $w_{\mathrm{d}}=u_{\mathrm{d}} t_{\text {cool }}$. Then, $E_{\max }$ and $B_{\mathrm{d}}$ are given by Eqs. (A.10) and (A.11), respectively. The right panel of Fig. 4 represents the allowed region of $E_{\max }$ and $B_{\mathrm{d}}$ when $w_{\mathrm{d}}=0.06-$ $0.4 \mathrm{pc}$ and $v_{\text {rolloff }}=(1.9-3.3) \times 10^{17} \mathrm{~Hz}$. All the points in the region satisfy other conditions. Vink \& Laming (2003) discussed similar arguments for Cas A.

\section{Discussion}

We have argued the limitations of the model of DSA from the recently observed scale length of the nonthermal X-ray emitting region of NE shell of SN 1006 based on the assumption that the observed spatial structure of nonthermal X-ray 

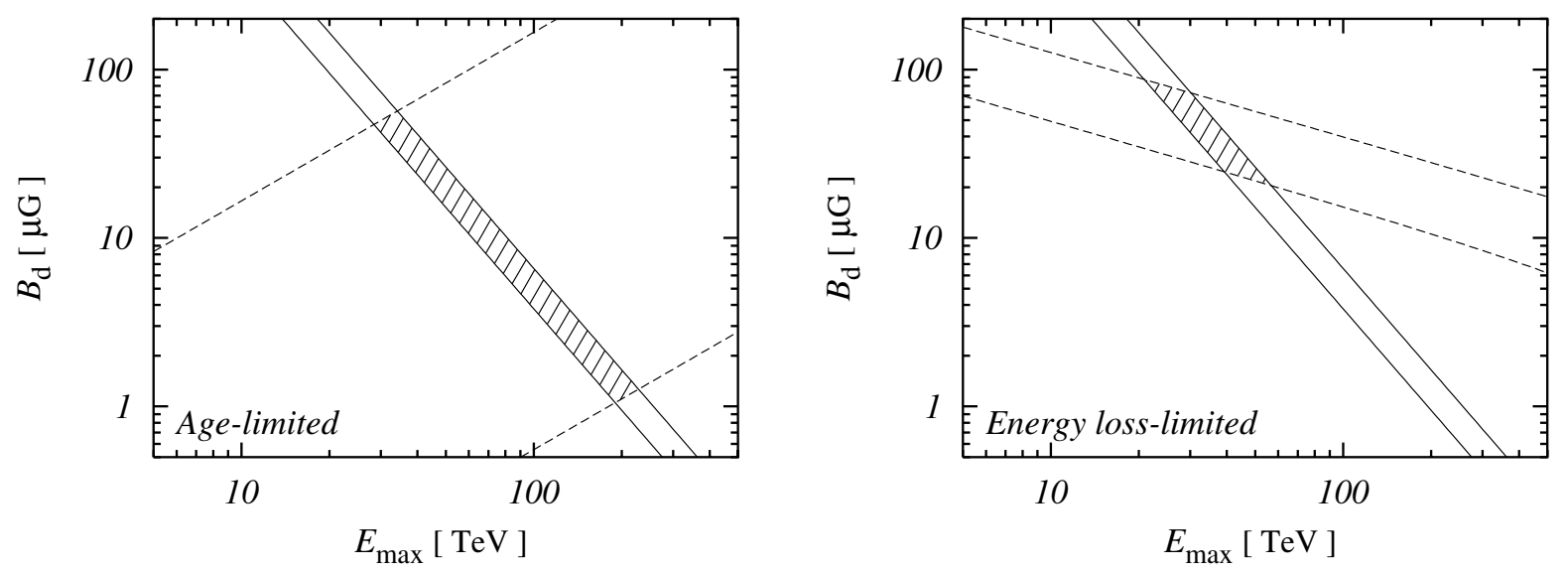

Fig. 4. The shaded areas indicate the most likely values of magnetic fields just behind the shock front $B_{\mathrm{d}}$ and the maximum energy of shockaccelerated electrons $E_{\max }$. The left panel is for the age-limited case, while the right gives the energy loss-limited model. These areas are formed by the two solid lines for a roll-off energy of $v_{\text {rolloff }}=(1.9-3.3) \times 10^{17} \mathrm{~Hz}$, and the two dashed lines from the observed width of nonthermal X-rays; in the age-limited case, a gyro-radius of accelerated electrons in the downstream should be $r_{\mathrm{g}}=E_{\max } /\left(\mathrm{e} B_{\mathrm{d}}\right)=(0.065-20) \times 10^{-2} \mathrm{pc}$, while in the energy loss-limited case, cooling time of accelerated electrons should be $t_{\text {cool }}=w_{\mathrm{d}} / u_{\mathrm{d}}=(0.81-5.4) \times 10^{2} \mathrm{yrs}$.

filaments reflects that of electrons accelerated to the maximum energy. A test particle approximation has been adopted, where the back reactions of accelerated particles are neglected, although one might have to consider corrections due to nonlinear effects to explain the wide-band spectrum from radio to $\mathrm{TeV} \gamma$ rays. Two models have been discussed: age-limited and energy loss-limited models. Note that in each model, the value and configuration of a magnetic field in the acceleration site can be discussed using only the spatial distribution of the synchrotron $\mathrm{X}$-rays, which are emitted by accelerated electrons with an energy of several tens of $\mathrm{TeV}$, and the roll-off frequency, which is derived by the fitting of the wide band spectrum from the radio to X-rays.

When a magnetic field is nearly parallel to the shock normal, $\eta_{\mathrm{u}}$ and $\eta_{\mathrm{d}}$ should be nearly unity, which means that the magnetic field is highly turbulent (near the Bohm limit), and the magnetic field is in the range of $20-85 \mu \mathrm{G}$. Relatively strong parallel magnetic fields are considered by several authors (Berezhko et al. 2002; Ellison et al. 2000). The derived value of the magnetic field seems to be higher than the usual interstellar value of a few micro Gauss. However, the mechanism proposed by Lucek \& Bell (2000) may be able to amplify the magnetic field. These situation can be realized both in the age-limited and energy loss-limited model.

When the magnetic field is nearly perpendicular to the shock normal, relatively small values of $B_{\mathrm{d}}=4 B_{\mathrm{u}}=14-20 \mu \mathrm{G}$ are allowed, which can exist only in the age-limited model. This value is consistent with the interstellar magnetic field and the value assumed in Bamba et al. (2003a) or others (Allen et al. 2001; Reynolds 1998) but slightly larger than that derived by CANGAROO observation (Tanimori et al. 1998, 2001), which assumes $\mathrm{TeV} \gamma$-rays are emitted by the IC process. This discrepancy may be solved if the filaments 1-6 are not the main sites of $\mathrm{TeV} \gamma$-rays generated by the leptonic process. The observed flux of $\mathrm{TeV} \gamma$-rays which are up-scattered $\mathrm{CMB}$ photons by synchrotron-emitting electrons is

$\mathcal{F}_{\text {IC }}=2.5 \times 10^{-2}\left(\frac{B_{\mathrm{d}}}{20 \mu \mathrm{G}}\right)^{-2} \mathcal{F}_{\text {synch }}$, where $\mathcal{F}_{\text {synch }}$ is the observed flux of synchrotron X-rays estimated as $1.8 \times 10^{-12}$ ergs s$~^{-1} \mathrm{~cm}^{-2}$ in the $0.5-10.0 \mathrm{keV}$ band by Bamba et al. (2003a). Then, the contribution of the IC $\gamma$-rays generated in the filaments $1-6$ is only $\sim 0.5 \%$ of the whole flux coming from SN 1006. This shows that the IC $\gamma$-ray emitting sites have a smaller magnetic field, hence radiate fewer synchrotron X-rays than in the filaments 1-6. Alternatively, the $\mathrm{TeV} \gamma$-rays may be emitted by the hadronic process. The most plausible position of the $\gamma$-ray emission determined by CANGAROO is north (and outside) of filament 1 (Tanimori et al. 1998, 2001). However, the region where the significance is higher than half the maximum value extends over $\sim 0.2^{\circ}$, which is almost the same as the standard deviation of the point-spread function of the CANGAROO telescope. We need stereoscopic observations of $\mathrm{TeV}$ gamma-ray emissions by imaging atmospheric Cherenkov telescopes (for example, CANGAROO-III, HEGRA, and H.E.S.S.) to establish the position and extent of the emission region.

We briefly discuss how our results may change if the test particle approximation is dropped. Although we should consider the spatial structure modified, for example, in the upstream precursor region, we present the cases of $r>4$ that are thought to be caused by nonlinear effects. Berezhko et al. (2002) showed that the present value of the total compression ratio is about 6 . In the age-limited case, we observe that the inferred magnitude of the upstream magnetic field becomes smaller than that of the $r=4$ case as $r$ is made larger (up to 7). However, the overall shapes and total areas of the allowed regions are changed only slightly for any case of $\theta$. Thus the nonlinear effect would not be large. On the other hand, in the energy loss-limited case, the allowed region in the $\eta_{\mathrm{u}}-$ $\eta_{\mathrm{d}}$ plane becomes narrow and only the cases of $\theta=0^{\circ}-10^{\circ}$ can be exist for $4<r \leqslant 6$ while no allowed region exists for $r \sim 7$. This comes from the fact that another restriction, Eq. (A.24), emerges. The larger $r$, the stronger the constraint because of the less efficient advection for a fixed shock velocity $u_{\mathrm{s}}$. Furthermore, it can be shown that there exists no case in which $w_{\mathrm{d}}$ is given by $\left(K_{\mathrm{d}} t_{\mathrm{cool}}\right)^{\frac{1}{2}}$, since the condition $t_{\mathrm{acc}}=t_{\text {cool }}$ 
is incompatible with the condition $u_{\mathrm{d}} t_{\text {cool }}<\left(K_{\mathrm{d}} t_{\text {cool }}\right)^{\frac{1}{2}}$ in the parameter range of interest.

It is important to determine the magnetic field configurations to discuss the acceleration and/or injection efficiency. In this paper, using the spatial distribution of nonthermal X-rays, we have shown that roughly two cases can exist: high and parallel, and low and perpendicular magnetic field. In the latter case, the back reaction of accelerated particles is small and thus a test-particle treatment is a good approximation. The magnetic field amplification process discussed by Lucek \& Bell (2000) does not work well. In the former case, nonlinear effects are so efficient that the magnetic field can be large. The difference between these cases probably comes from the fact that the (proton) injection rate depends strongly on the shock obliquity and diminishes as $\theta$ increases (Völk et al. 2003). In addition to our result, radio polarization data with high spatial resolution may provide further information about the magnetic field configuration.

In this paper, we have adopted the plane shock approximation. For further details, it might be important to consider the curvature effect as discussed in Berezhko et al. (2003) to produce a more realistic scenario.

Acknowledgements. Our particular thanks are due to T. Nakamura, M. Hoshino, and T. Tanimori for their fruitful discussions and comments. R.Y. and A.B. are supported by JSPS Research Fellowships for Young Scientists. This work is supported by a Grant-in-Aid for Scientific Research, No. 14340066 from the Ministry of Education, Culture, Sport, Science, and Technology of Japan, and also supported by a Grant-in-Aid for for the 21st Century COE "Center for Diversity and Universality in Physics".

\section{Appendix A: Detailed calculations}

We summarize a procedure for practical calculation of $\eta_{\mathrm{u}}$ and $\eta_{d}$ from observed quantities. Once $(f, g)$ is given for fixed $\theta$, the allowed region of $\left(\eta_{\mathrm{u}}, \eta_{\mathrm{d}}\right)$ is derived by Eqs. (8) and (9). Relations of $f$ and $g$ in the age-limited case and energy losslimited case are different.

\section{A.1. Age-limited case}

One can derive from Eqs. (12) and (13)

$\frac{g}{f}=r^{-1} \frac{w_{\mathrm{d}}}{w_{\mathrm{u}}}$

The condition $t_{\mathrm{acc}} \lesssim t_{\text {loss }}$ gives the additional inequalities. Substituting Eqs. (6) and (7) into Eq. (2), we derive

$t_{\mathrm{acc}}=4 \phi(f+r g) \frac{c E_{\mathrm{max}}}{3 \mathrm{e} B_{\mathrm{d}} u_{\mathrm{s}}^{2}}$.

Where $\phi=\phi(r)=(3 / 4) r /(r-1)$. The energy loss timescale that should be compared with $t_{\text {acc }}$ is given by

$t_{\text {loss }}=\frac{6 \pi m_{\mathrm{e}}^{2} c^{3}}{\sigma_{T} E_{\max } B_{\mathrm{d}}^{2}} \frac{f+r g}{R^{-2} f+r g}$.
Using Eq. (1), we can eliminate $E_{\max }$ and $B_{\mathrm{d}}$, and obtain

$$
\begin{aligned}
R(\theta)^{-2} f+r g \lesssim & 4.8 \phi^{-1}\left(\frac{v_{\text {rolloff }}}{2.6 \times 10^{17} \mathrm{~Hz}}\right)^{-1} \\
& \times\left(\frac{u_{\mathrm{s}}}{2.89 \times 10^{8} \mathrm{~cm} \mathrm{~s}^{-1}}\right)^{2}
\end{aligned}
$$

The quantities $f$ and $g$ should satisfy Eqs. (A.1) and (A.4).

The condition $t_{\mathrm{acc}} \lesssim t_{\text {loss }}$ gives also the upper limit of $B_{\mathrm{d}}$. In the age-limited case, one can derive $\chi=\left(R^{-2} w_{\mathrm{u}}+w_{\mathrm{d}}\right) /\left(w_{\mathrm{u}}+w_{\mathrm{d}}\right)$. Then using Eqs. (10) and (14), we derive

$$
\begin{aligned}
B_{\mathrm{d}} \lesssim & 30 \mu \mathrm{G} \phi^{-2 / 3}\left(\frac{v_{\text {rolloff }}}{2.6 \times 10^{17} \mathrm{~Hz}}\right)^{-1 / 3} \\
& \times\left(\frac{w_{\mathrm{u}} R^{-2}(\theta)+w_{\mathrm{d}}}{0.25 \mathrm{pc}}\right)^{-2 / 3}\left(\frac{u_{\mathrm{s}}}{2.89 \times 10^{8} \mathrm{~cm} \mathrm{~s}^{-1}}\right)^{2 / 3}
\end{aligned}
$$

where we eliminate $E_{\max }$ with Eq. (1).

Additionally, using Eqs. (1) and (13), one can show $E_{\max }$ and $B_{\mathrm{d}}$ can be expressed in terms of $g$ as

$$
\begin{aligned}
E_{\max }= & 41 \mathrm{TeV}\left(\frac{r}{4}\right)^{-1 / 3}\left(\frac{v_{\text {rolloff }}}{2.6 \times 10^{17} \mathrm{~Hz}}\right)^{1 / 3} \\
& \times\left(\frac{g^{-1} w_{\mathrm{d}}}{0.2 \mathrm{pc}}\right)^{1 / 3}\left(\frac{u_{\mathrm{s}}}{2.89 \times 10^{8} \mathrm{~cm} \mathrm{~s}^{-1}}\right)^{1 / 3} \\
B_{\mathrm{d}}= & 31 \mu \mathrm{G}\left(\frac{r}{4}\right)^{2 / 3}\left(\frac{v_{\text {rolloff }}}{2.6 \times 10^{17} \mathrm{~Hz}}\right)^{1 / 3} \\
& \times\left(\frac{g^{-1} w_{\mathrm{d}}}{0.2 \mathrm{pc}}\right)^{-2 / 3}\left(\frac{u_{\mathrm{s}}}{2.89 \times 10^{8} \mathrm{~cm} \mathrm{~s}^{-1}}\right)^{-2 / 3}
\end{aligned}
$$

respectively.

\section{A.2. Energy loss-limited case}

For our parameters, $w_{\mathrm{u}}$ and $w_{\mathrm{d}}$ are given by

$w_{\mathrm{u}}=K_{\mathrm{u}} / u_{\mathrm{s}}$

$w_{\mathrm{d}}=u_{\mathrm{d}} t_{\mathrm{cool}}$

We can calculate $E_{\max }$ and $B_{\mathrm{d}}$ from Eqs. (1) and (A.9) as

$$
\begin{aligned}
E_{\max }= & 39 \mathrm{TeV}\left(\frac{r}{4}\right)^{1 / 3}\left(\frac{v_{\text {rolloff }}}{2.6 \times 10^{17} \mathrm{~Hz}}\right)^{2 / 3} \\
& \times\left(\frac{w_{\mathrm{d}}}{0.2 \mathrm{pc}}\right)^{1 / 3}\left(\frac{u_{\mathrm{s}}}{2.89 \times 10^{8} \mathrm{~cm} \mathrm{~s}^{-1}}\right)^{-1 / 3},
\end{aligned}
$$

$$
\begin{aligned}
B_{\mathrm{d}}= & 35 \mu \mathrm{G}\left(\frac{r}{4}\right)^{-2 / 3}\left(\frac{v_{\text {rolloff }}}{2.6 \times 10^{17} \mathrm{~Hz}}\right)^{-1 / 3} \\
& \times\left(\frac{w_{\mathrm{d}}}{0.2 \mathrm{pc}}\right)^{-2 / 3}\left(\frac{u_{\mathrm{s}}}{2.89 \times 10^{8} \mathrm{~cm} \mathrm{~s}^{-1}}\right)^{2 / 3}
\end{aligned}
$$

Then, from Eqs. (6) and (A.8), we derive

$$
\begin{aligned}
f= & 4.8 \frac{w_{\mathrm{u}}}{w_{\mathrm{d}}}\left(\frac{r}{4}\right)^{-1}\left(\frac{v_{\text {rolloff }}}{2.6 \times 10^{17} \mathrm{~Hz}}\right)^{-1} \\
& \times\left(\frac{u_{\mathrm{s}}}{2.89 \times 10^{8} \mathrm{~cm} \mathrm{~s}^{-1}}\right)^{2} .
\end{aligned}
$$


The condition $t_{\mathrm{acc}}=t_{\text {loss }}$ gives

$$
\begin{aligned}
R(\theta)^{-2} f+r g= & 4.8 \phi^{-1}\left(\frac{v_{\text {rolloff }}}{2.6 \times 10^{17} \mathrm{~Hz}}\right)^{-1} \\
& \times\left(\frac{u_{\mathrm{s}}}{2.89 \times 10^{8} \mathrm{~cm} \mathrm{~s}^{-1}}\right)^{2}
\end{aligned}
$$

Eliminating $v_{\text {rolloff }}$ from Eqs (A.12) and (A.13), we derive

$\frac{g}{f}=\frac{1}{4}\left[\phi^{-1} \frac{w_{\mathrm{d}}}{w_{\mathrm{u}}}-\left(\frac{r}{4}\right)^{-1} R(\theta)^{-2}\right]$.

Equations (A.13) and (A.14) determine $(f, g)$.

We can show that the condition $t_{\text {loss }}<t_{\text {age }}$ is always satisfied. Substituting Eqs. (A.10) and (A.11) into Eq. (A.3), we obtain

$$
\begin{aligned}
t_{\mathrm{loss}}= & 2.7 \times 10^{2} \mathrm{yrs}\left(\frac{r}{4}\right)\left(\frac{w_{\mathrm{d}}}{0.2 \mathrm{pc}}\right) \\
& \times\left(\frac{u_{\mathrm{s}}}{2.89 \times 10^{8} \mathrm{~cm} \mathrm{~s}^{-1}}\right)^{-1} \frac{f+r g}{R^{-2} f+r g} .
\end{aligned}
$$

Then, $t_{\text {loss }}<t_{\text {age }}$ reduces

$$
\begin{aligned}
\frac{1+r g / f}{R^{-2}+r g / f}< & 3.7\left(\frac{r}{4}\right)^{-1}\left(\frac{w_{\mathrm{d}}}{0.2 \mathrm{pc}}\right)^{-1} \\
& \times\left(\frac{u_{\mathrm{s}}}{2.89 \times 10^{8} \mathrm{~cm} \mathrm{~s}^{-1}}\right)\left(\frac{t_{\mathrm{age}}}{1000 \mathrm{yrs}}\right) .
\end{aligned}
$$

Using Eq. (A.14), we rewrite the left hand side of Eq. (A.16) as

$\frac{1+r g / f}{R^{-2}+r g / f}=1+\left(1-R(\theta)^{-2}\right) \phi\left(\frac{r}{4}\right)^{-1} \frac{w_{\mathrm{u}}}{w_{\mathrm{d}}}$.

Substituting Eq. (A.17) into Eq. (A.16), we derive

$$
\begin{aligned}
w_{\mathrm{d}}+ & \left(1-R(\theta)^{-2}\right) \phi\left(\frac{r}{4}\right)^{-1} w_{\mathrm{u}}<0.74 \mathrm{pc}\left(\frac{r}{4}\right)^{-1} \\
& \times\left(\frac{u_{\mathrm{s}}}{2.89 \times 10^{8} \mathrm{~cm} \mathrm{~s}^{-1}}\right) \times\left(\frac{t_{\mathrm{age}}}{1000 \mathrm{yrs}}\right) .
\end{aligned}
$$

This equation is always satisfied since $w_{\mathrm{u}}$ and $w_{\mathrm{d}}$ range between $0.01-0.1 \mathrm{pc}$ and $0.06-0.4 \mathrm{pc}$, respectively.

Finally in order to validate Eqs. (A.8) and (A.9), we confirm the conditions

$$
\begin{aligned}
& K_{\mathrm{u}} / u_{\mathrm{s}}<\left(K_{\mathrm{u}} t_{\text {cool }}\right)^{\frac{1}{2}}, \\
& u_{\mathrm{d}} t_{\text {cool }}>\left(K_{\mathrm{d}} t_{\text {cool }}\right)^{\frac{1}{2}},
\end{aligned}
$$

are always satisfied for our parameters of interest. Using Eqs. (1), (6) and (10), Eq. (A.19) can be rewritten as

$f<19.0\left(\frac{u_{\mathrm{s}}}{2.89 \times 10^{8} \mathrm{~cm} \mathrm{~s}^{-1}}\right)^{2}\left(\frac{v_{\text {rolloff }}}{2.6 \times 10^{17} \mathrm{~Hz}}\right)^{-1}$.

This equation, together with Eq. (A.12), reduces to

$$
\frac{w_{\mathrm{u}}}{w_{\mathrm{d}}}<r
$$

which is always satisfied since $w_{\mathrm{u}}=0.01-0.1 \mathrm{pc}$ and $w_{\mathrm{d}}=$ 0.06-0.4 pc. On the other hand, from Eqs. (1), (7), (10), and (A.20), we derive

$g<1.2\left(\frac{r}{4}\right)^{-2}\left(\frac{u_{\mathrm{s}}}{2.89 \times 10^{8} \mathrm{~cm} \mathrm{~s}^{-1}}\right)^{2}\left(\frac{v_{\text {rolloff }}}{2.6 \times 10^{17} \mathrm{~Hz}}\right)^{-1}$.

Combining this equation and Eq. (A.13), we eliminate $v_{\text {rolloff }}$ and $u_{\mathrm{s}}$ as

$\left[1-\left(\frac{r}{4}\right)^{-1} \phi\right] g<\frac{1}{4}\left(\frac{r}{4}\right)^{-2} \phi R(\theta)^{-2} f$.

As long as $r \leq 4$, this is always satisfied since $1-(r / 4)^{-1} \phi=$ $(r-4) /(r-1) \leq 0$.

\section{References}

Achterberg, A., Blandford, R. D., \& Reynolds, S. P. 1994, A\&A, 281, 220

Aharonian, F. A., \& Atoyan, A. M. 1999, A\&A, 351, 330

Allen, G. E., Petre, R., \& Gotthelf, E. V. 2001, ApJ, 558, 739

Bamba, A., Yamazaki, R., Ueno, M., \& Koyama, K. 2003a, ApJ, 589, 827

Bamba, A., Yamazaki, R., Ueno, M., \& Koyama, K. 2003b, Adv. Space Res., in press [astro-ph/0308322]

Bell, A. R. 1978, MNRAS, 182, 443

Berezhko, E. G., Ksenofontov, L. T., \& Völk, H. J. 2002, A\&A, 395, 943

Berezhko, E. G., Ksenofontov, L. T. \& Völk, H. J. 2003, in Proc. of 28th ICRC, 2441

Blandford, R. D., \& Eichler, D. 1987, Phys. Rep., 154,1

Blandford, R. D., \& Ostriker, J. P. 1978, ApJ, 221, L29

Drury, L. O’ C. 1983, Rep. Prog. Phys., 46, 973

Dyer, K. K., Reynolds, S. P., Borkowski, K. J., Allen, G. E., \& Petre, R. 2001, ApJ, 551, 439

Ellison, D. C., Berezhko, E. G., \& Baring, M. G. 2000, ApJ, 540, 292

Ghavamian, P., Winkler, P. F., Raymond, J. C., \& Long, K. S. 2002, ApJ, 572, 888

Jones, F. C., \& Ellison, D. C. 1991, Space Sci. Rev., 58, 259

Jokipii, J. R. 1987, ApJ, 313, 842

Koyama, K., Petre, R., Gotthelf, E. V., et al. 1995, Nature, 378, 255

Long, K. S., Reynolds, S. P., Raymond, J. C., et al. 2003, ApJ, 586, 1162

Lucek, S. G., \& Bell, A. R. 2000, MNRAS, 314, 65

Malkov, E., \& Drury, L. O’C. 2001, Rep. Prog. Phys., 64, 429

Ratkiewicz, R., Axford, W. I., \& McKenzie, J. F. 1994, A\&A, 291, 935

Reynolds, S. P. 1998, ApJ, 493, 375

Reynolds, S. P., \& Keohane, J. W. 1999, ApJ, 525, 368

Skilling, J. 1975, MNRAS, 172, 557

Tanimori, T., Hayami, Y., Kamei, S., et al. 1998, ApJ, 497, L25

Tanimori, T., Naito, T., Yoshida, T., \& CANGAROO collaboration 2001, in Proc. of 27th ICRC (Hamburg), 2465

Vink, J., \& Laming, J. M. 2003, ApJ, 584, 758

Völk, H. J., Berezhko, E. G., \& Ksenofontov, L. T. 2003, A\&A, 409, 563

Winkler, P. F., Gupta, G., \& Long, K. S. 2003, ApJ, 585, 324 